\title{
Urban Design of Historic districts Based on Action Planning
}

\author{
Wang Yanjun ${ }^{a,}{ }^{*}$, He Jianxiong \\ College of Urban and Environmental Sciences, Northwest University, Xi'an 710127, China. \\ a974702583@qq.com
}

\begin{abstract}
Historical districts are faced with problems of inadequate features protection and landscape destruction in the process of urban development. At the present stage, the urban design of historical districts has a series of problems, like inadequate expression of characteristics incomplete framework and the lack of transformation route from design to implementation. Urban design based on action planning is action-oriented with characteristics of comprehensive planning, complete workflow and clear implementation projects, which is a new attempt for urban design of historical districts. Urban design of Xi'an Shuncheng Lane used action planning as a concept, established an framework including "current status and problems — target location and strategy_action project and timing-achievement improvement and evaluation", to achieve effective implementation in historical districts.
\end{abstract}

Keywords: Action planning, urban design, North Shuncheng lane

\section{Introduction}

Urban design plays an important role in ensuring the implementation of urban planning and in enhancing the level of urban construction. In order to improve the urban competitiveness, large-scale urban design has been carried out all over China. As an important carrier to extend urban context, historic districts are facing problems of destruction and inadequate protection in the process of rapid urban development, it is imperative to apply urban design to realize the protection and update of historic districts. Although the contents of urban design in historic districts are similar to those in urban areas, it not only pay attention to building entity and street environment that form the material space, but also put more emphasis on the expression of social factors, including the inheritance of traditional features, the combination of historical context and modern life etc. [1]. In Western developed countries, urban design is used to protect historic districts and their landscape features by identifying landscape elements and spatial patterns with type method, parameter method, etc. [2-4]. In China urban design is carried out in the reconstruction of architecture elevation or newly built districts, update methods include organic updates, function updates, etc. [5-7]. In summary, the number of urban design continues to increase, but the immature standard and the incomplete procedure lead to a disconnection between the implementation and the setting goals, which make the achievements cannot effectively guide the development of historic districts [8].

The reason for this problem lies in the gap between the setting goals and the implementation route of urban design [9]. Main problems exist in urban design are the following: firstly, inadequate expression of characteristics. Urban design at present pays too much attention to the form of material space, and lacks the research of the comprehensive characteristics and future needs of the particular districts. Secondly, imperfect workflow. The emphasis of different stages is not clear, which causes the final results are tedious. Thirdly, the lack of implementation route from plans to implementation. Traditional urban design uses diagrams to guide the implementation of each element, and lacks the analyses on concrete projects.

Therefore, it is necessary to establish an effective implementation route between urban design and design objects to realize the effective implementation in planning practice. "Action planning” is 
defined as "the implementation-oriented planning" [10], which proposes specific projects that can be implemented in the near future based on the principle of convenient for implement and maximization of comprehensive benefits [11]. Practice of action planning in China is mainly used in overall urban design, town planning and comprehensive urban planning, its application in historic districts urban design is relatively less. This paper will take North Shuncheng Lane of Xi'an city as an example to practice the urban design based on action planning. The purpose of this study is to establish a new method of conservation and update historic districts based on action-orientation.

\section{Urban design of historic districts based on action planning}

The emphasis of urban design in historic districts with action planning lies in the analysis of the demand of future development and steps of action projects. The correct positioning of future development needs to be combined with a deep understanding of historic districts' social, economic, ecological, cultural conditions and its status in urban development. Action projects should take the feasibility of each project and the synergy between different projects into consideration, and adjust the action strategy according to the external environment and the change of district itself.

The framework can be divided into four stages (Figure 1). (1) Preparation stage includes current status analysis and thematic studies. Urban design analyzes thoroughly which not only includes the basic situations such as the historical evolution of the city and the status of the districts in the urban development, but also includes the material aspects such as the use of the land, the status of buildings, and other non-material aspects such as the history of the districts, spirit culture and folkways culture. (2) Planning stage includes the target location and development strategy. Urban design with action planning determines development goals from the interests of the districts' internal power and the city's external thrust. It proposes a comprehensive development strategy from space, industry, lifestyle, ecology etc. on the purpose of achieving the overall goal by achieving each part's sub goal. (3) Implementation stage includes the arrangement and implementation of specific projects. Urban design with action planning is under the guidance of realizing the best interests of districts to arrange action projects such as industrial planning, public space planning, cultural planning, and defines project subjects, project sequences and project types. (4) Evaluation stage includes achievement improvement and evaluation. Urban design with action planning is a dynamic and long-term planning, which will timely summarize the implementation and sustainability of projects. Through the establishment of mission system, compilation system and evaluation system, it provides scientific guidance for the policy environment.

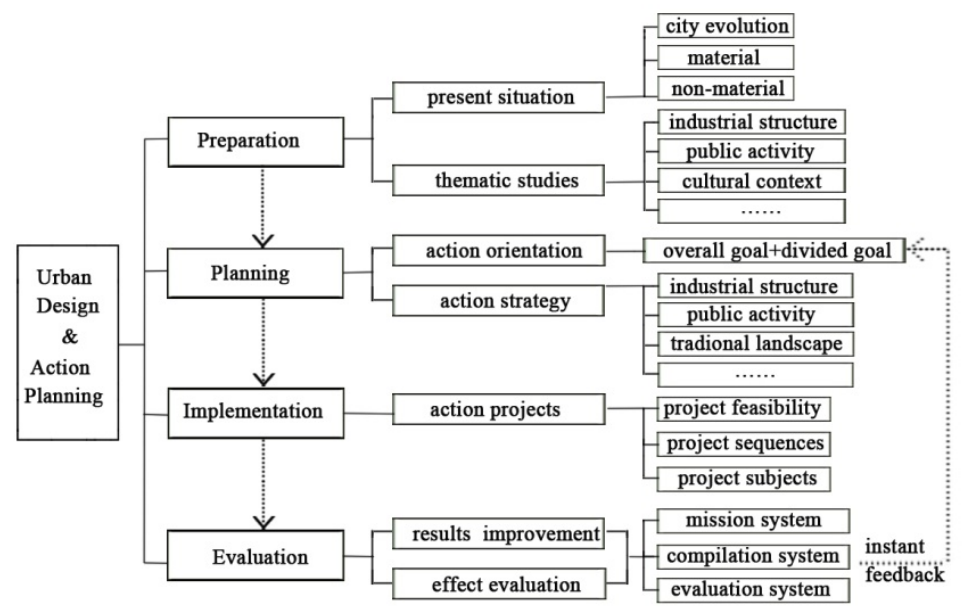

Fig. 1 Technology roadmap for urban design of historic districts based on action planning 
Introducing the concept of action planning into urban design of historic districts helps to solve the existing problems such as the inadequate expression of characteristics, incomplete framework and the lack of transformation route. Urban design of historic districts under action planning takes into account the current situation comprehensively including economy, society, culture etc., arranges specific projects includes basic analysis, target position, action strategy, action projects and effect evaluation. Action planning points out the ultimate objectives, main participants and time arrangement, and gradually perfects the planning results in the dynamic process.

\section{Urban design of North Shuncheng Lane based on action planning}

\subsection{Current status and problems}

North Shuncheng Lane is located in the northern of Ming city wall in the center of Xi'an City. It's about 950 meters long and covers a total area of about $20 \mathrm{hm}^{2}$.It can be divided into two parts by middle road, residence pattern in the north has the level of street, lane, courtyard and neighborhood,. residence pattern in the south are mostly plate-like unit buildings.

Through research on thematic studies, we can find that in space quality, the indiscriminate construction and the lack of infrastructure makes living quality decline. In industrial structure, the original features such as leisure and recreation are in a gradual degradation. In cultural context, the damaged landscape cannot afford the function of continuing historical context, and the intangible cultural heritage is disappearing. In ecological construction, residents formed a series of adaptive greening ways such as wall greening and potted plants, but the waste along the street erode the public space of the streets.

\subsection{Target location and strategy}

The target of urban design should focus on how to improve living quality and enhance the vitality, with maintaining historical and cultural context. Facing the problems above, urban design starts with the relationship of slow traffic system and public traffic system, the relationship between urban function and space demand, the relationship between leisure life and landscape environment, the utilization of old buildings, and breaks down the setting goals into four sub goals as slow travel, leisurely life, contextual continuity and green ecology.

The four sub goals will be transformed equivalently into five action strategies, which are optimizing traffic system, improving traditional landscape, developing industrial structure, guiding public space and advocating low-carbon lifestyle (Figure 2).

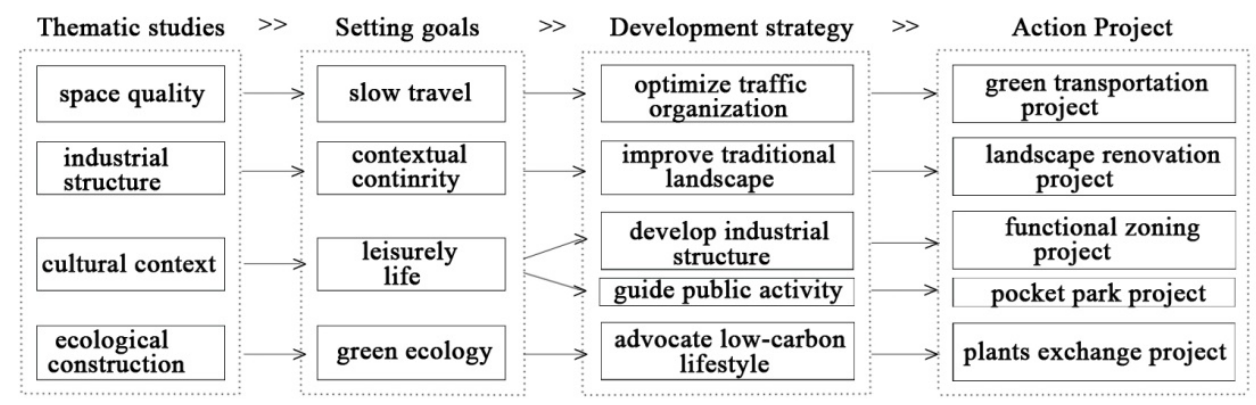

Fig.2 Flow chart of urban design in North Shuncheng Lane based on action planning

\subsection{Action project and timing}

Action strategies are carried out as specific action projects, including green transportation project, landscape renovation project, functional zoning project, pocket park project and plants exchange project.

"Green transportation project" aims at optimizing traffic system. Firstly, it creates slow-moving 
system by using symbolic significance like bricks, woods and stones to reflect the cultural features,. It also sets up tour routes for tourists along middle road with historical story drawing on the wall. Secondly, it organizes one-way traffic. This project sets western Lane and the central north-south road as one-way road in the opposite direction, making use of secondary roads with high density but low utilization to evacuate traffic congestion on the main roads.

"Landscape renovation project" aims at restoring the street scene from block scale and building scale. In block scale, using traditional form of narrow width and deep depth, it forms the living form of "shop underneath and housing above", creating a traditional atmosphere. In building scale, it mainly updates the building facade with the connected but not continuous architectural interface of corridors and brick paving, and reconstructs roofs with double slope or single slope. (Figure 5).

"Functional zoning project" aims at developing industrial structure. In the principles of restoring the traditional unit, traditional street space and traditional folk culture, it optimizes the functional layout based on its original functions such as leisure, entertainment and services. Creative Workshop with traditional handicraft shops allows visitors to experience the process of making clay, paper-cut and other traditional crafts. Green Workshop encourages residents to replace waste into plants, allowing visitors to experience the process of waste reuse. Artists Workshop transforms the abandoned factory into space for communication of the artists, allowing visitors to experience the production process of arts. Courtyard Workshop introduces conceptual hotels with Guanzhong courtyard as the prototype.

"Pocket park project" aims at promoting the quality of leisure. This project uses the corner space to create a "pocket park", which is a small-scaled open space for various theme activities, and scatters in a patchy form in the block. It plans different types of theme activities based on different places, such as game angle, welcoming pavilion, and connects by walking routes to highlight neighborhood characteristics.

"Plants exchange project" aims at advocating low-carbon lifestyle. This project sets up an exchange platform for waste and plant, and selects rooms for raw materials and gardening in Green Workshop. Residents' actions call on residents to classify waste and send to Green Workshop, staffs provide potted plants of equal value and encourage residents to place them in public places for tourists to appreciate. Tourists' actions allow tourists to use recycled waste such as plastic bottles as raw materials, and experience the production process of gardening, staffs encourage tourists to place the potted plants in the display area for the next round of exchange.

\subsection{Achievement improvement and evaluation}

The system of action mission, compilation and evaluation improves the effectiveness of urban design. A tightly connected system of action mission is established to make explicit demand for the subject in different sages. A multi-level interactive system of compilation is established to invite experts to evaluate the implementation and to encourage public to put forward suggestions for the design contents. A supervision system with regular inspection is established to urge departments to review the implementation of action projects and to provide a realistic basis for the next amendment.

\section{Conclusion and discussion}

In view of the problems existing in the urban design of historic districts, urban design based on action planning has the characteristics of comprehensive planning, complete workflow and clear implementation projects,. Under this concept, the urban design of Shuncheng Lane in Xi'an, based on the research of the present situations including space, industries, culture and ecology etc., puts 
forward the sub goals of slow travel, leisurely life, contextual continuity and green ecology, and constructs the action strategies of optimizing traffic system, improving traditional landscape, developing industrial structure, guiding public space and advocating low-carbon lifestyle. These strategies are translated into concrete action projects like green transportation project, landscape renovation project, pocket park project, plants exchange project, functional zoning project. Through "green transportation project", the resources are effectively integrated by dredging the inner network of the districts and planning tour routes with the theme of Xi'an city wall. Through "pocket park project" and "plants exchange project", different types of activities are arranged for different public spaces, and a platform for the exchange of plants and recyclable waste is set up. Through "functional zoning project", the traditional services transform into tourism services and handicraft industries.

As an important means of protection of historic districts, urban design should pay more attention to the methods of how to implement design ideas to improve the operability. Through specific action strategy and action project, urban design based on action planning concerns the implementation of the entire processes, which will be of great significance to improve the urban design system. In the future, action planning can be considered to explore into legal system, to realize the standardization and institutionalization in terms of design processes, implementation and evaluation, and to promote regional development orderly.

\section{Acknowledgments}

This work was financially supported by National Science and Technology Support Program (2015BAL01B04) and Natural Science Foundation of Shaanxi Province (2015JM5171)..

\section{References}

[1] Ruan Yisan, Sun Meng. The study on some issues related to the conservation and planning for the historic streets and areas in china, J. City Planning Review. 25(2001) 25-32.

[2] Wu Xiaosong, Zhang Ying, Wu Lv. Town planning system evolution in England since the 20th century. J. Urban Planning International. 24 (2009) 45-50.

[3] Gu Dazhi, Guan Zaolin. “Dynamic planning” theory and practices in UK. J. Urban Planning. 37 (2013) 81-88.

[4] Tiesdell.S, Heath.T, Taner.O.C. The revival of urban historic blocks. M. Beijing: China Architecture and Building Press, 2006.

[5] Huo Jun, Han Rong. The function replacement of urban historic district under the experience economy. J. Urban Problems.3(2013) 28-32.

[6] Wu Liangyong, Wu Tinghai. From strategy plan to action plan.J. Urban Planning. 27(2003) 13-17.

[7] Luo Yong. Effective Approach towards Plan-making and Implementation of Action Plan.J. Urban Development Studies.21(2014)8-11.

[8] Zhang Yongmei.Action Planning Concept Based Comprehensive Urban Design Approach: Anji Case.J. Planners. 28(2012)42-45.

[9] Zhao Yongwei, Ye Weihua. Methods for comprehensive urban design implementation. J. Planners, 26(2010)15-19.

[10] Stone C. Regime Polities: Governing Atlanta, 1946-1988. M. University Press of Kamas, 1989. [11] Wang Hong. Action plan and its implications to planning practice in China. J. City Planning Review. 29(2005)41-46. 